\title{
Electron-hole Fermi liquid in nanosized semiconductor structures
}

\author{
V.G. Litovchenko, A.A. Grygoriev \\ V. Lashkaryov Institute of Semiconductor Physics, NAS of Ukraine, \\ 41, prospect Nauky, 03028 Kyiv, Ukraine; e-mail: lvg@isp.kiev.ua
}

\begin{abstract}
The experimental and theoretical results on the quantum-sized electron-hole liquid plasma (EHLP) in semiconductors and analysis of the difference of it in comparison to the bulk one have been presented. The non-equilibrium Fermi EHLP can be created in the bulk and layered structures (insulator-semiconductor interfaces, thin films, quantum superlattices, etc.) at low temperatures and powerful laser radiation. In the quantum-sized structures, however, these phenomena appear at much higher temperatures, up to the room ones. The peculiarities of EHLP phenomena are: (1) appearance the very broad luminescence line in the low-energy side of its spectrum, which have constant width and energy position under variation of the light intensity as well as narrowing peak when increasing the temperature; (2) appearance of stimulated radiation with a relatively low excitation threshold (the so-called "surface laser effect"); (3) planar ballistic expansion of electron-hole plasma over long distances; (4) predicted effect of transformation of non-equilibrium $2 \mathrm{D}$ plasmons into radiative modes.
\end{abstract}

Keywords: electron-hole plasma, quantum-sized structures, luminescence.

Manuscript received 19.10.09; accepted for publication 22.10.09; published online 04.12.09.

\section{Introduction}

At first, the electron-hole Fermi liquid plasma (EHLP) was found and studied in bulk semiconductors as a result of large exciton concentration $n_{c r}$ at high excitation levels as well as at low temperatures [1-5]. It was a new specific low-temperature liquid matter consisted of electron-hole (e-h) quasi-neutral pairs.

The reason for appearance of EHLP is rather large e-h interaction at short distances between neutral excitons that have rather large Bohr radius $r_{B}=\left(\frac{\hbar^{2}}{e^{2}}\left(\frac{\varepsilon}{m^{*}}\right)\right)$ and moderate Coulomb band energy in semiconductor matter

$$
E_{c r}=\frac{e^{4}}{2 \hbar^{2}}\left(\frac{m^{\alpha}}{\varepsilon}\right)=\left(\frac{e^{2}}{2 \varepsilon}\right) \frac{1}{r_{B}}
$$

Due to the typically (for semiconductors) large values of dielectric constants $\varepsilon \geq 10$ and small values of the effective mass of electrons $m_{e} / m_{0}<<1$ and holes $m_{h}$, this predicts relatively small concentration of the eh pairs: $n_{c r} \sim\left(r_{B}\right)^{-3}$ for 3D case, $n_{c r} \sim\left(r_{B}\right)^{-2}$ for $2 \mathrm{D}$, and $n_{c r} \sim\left(r_{B}\right)^{-1}$ for $1 \mathrm{D}$ case.
The interest to this phenomenon is strongly increasing now [6-8] due to perspectives in using the EHLP for observation of such principal effects as transport of e-h pairs, coupling energy, generation of the $\mathrm{THz}$ microwaves, laser irradiation in nanostructures etc. Below we will present the results for low-size structures of different semiconductors.

\section{Theoretical analysis of EHLP properties of in the quantum-sized case}

As follows from the theory of collective interactions between electrons and holes developed earlier [9-12], the appearance of the Fermi EHLP is explained by the fact, that, owing to many-particle exchange and correlation interactions of electrons and holes, the binding energy per pair of particles in this liquid is higher than in exciton gas. The condensation energy $E_{c}$ can be expressed as:

$$
E_{c}=E_{\text {kin }}-E_{\text {Coul }}=E_{F}-\left(E_{\text {cor }}+E_{\text {exch }}\right) \text {. }
$$

The quantities of the kinetic (anti-bonding) energy (Fermi energy) as well as bonding Coulomb correlation and exchange $E_{\text {exch }}$ bonding energy appear in the relation (2) with opposite signs (Fig. 1). The ideal gas transforms into a system of interacting quasi-particles (excitons) at a fairly high exciton density $n_{c r} \geq r_{e x}^{-3}$ with the threshold concentration corresponding to the value given by 
$n_{c r}=4\left(\frac{m^{*} k T}{2 \pi \hbar^{2}}\right)^{3 / 2} \exp \left(\frac{E_{n}+E_{p}-E_{e x}}{k T}\right)$

Here $E_{n}, E_{p}$ are the Fermi quasi-levels of electrons and holes, respectively, and $E_{e x}$ denotes the exciton binding energy. This liquid contains neither excitons nor their "molecules", but does density of e-h plasma resemble a liquid atomic metal, such as a light alkali. To see this difference, we should mention that a molecular liquid is formed because the intramolecular interaction greatly exceeds the intermolecular value. For an exciton molecule this is not true, here the quantities mentioned are of the same order, and the magnitude is less since there is no heavy nucleus to confine a lighter component. In fact, the dissociation energy of a two-particle molecule, where $\omega_{0}$ is zero point energy. As long as $\omega_{0} \sim 1 / \sqrt{M}$ then with decreasing of $M$ (nuclear mass) the value $\frac{\hbar \omega_{0}}{2} \rightarrow U_{\min }$, hence $E_{d i s} \rightarrow 0$, i.e. "electron molecule" practically cannot be formed.
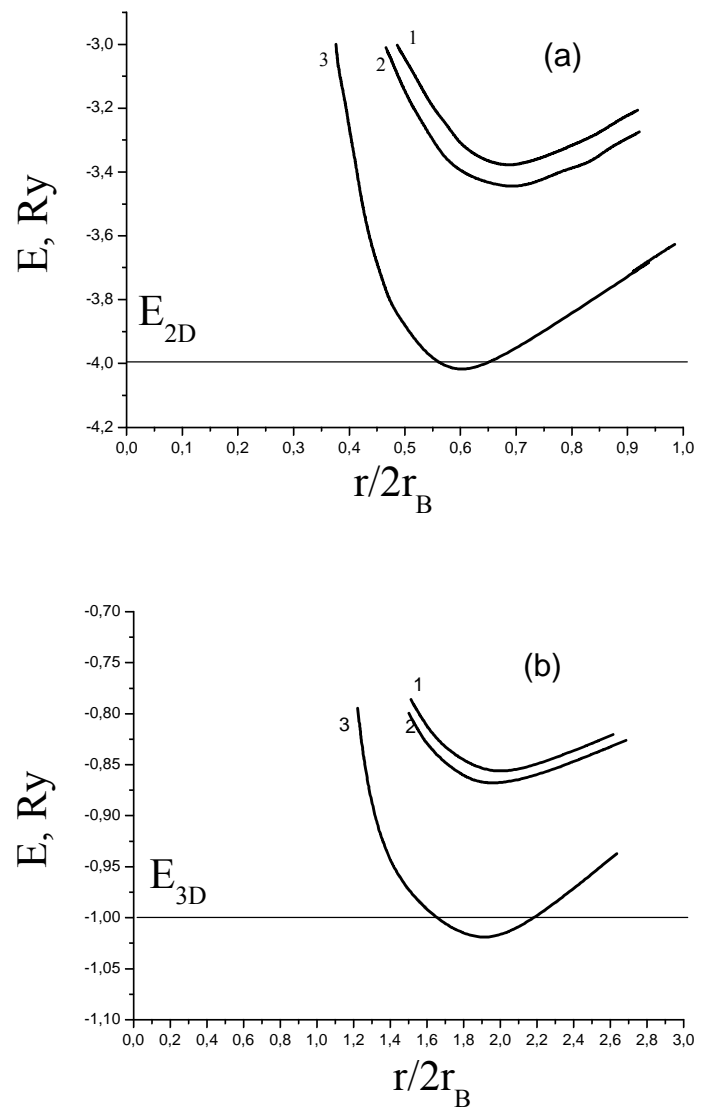

Fig. 1. Ground state energy $E_{c}$ (condensation energy) versus $r$ for various values $m_{h} / m_{e}=1,1.5$, and 10 (curves $1,2,3$, respectively) in the case of a single valley in the c-band Dashed line: binding energy for 2D (left) and 3D (right) excitons [13].
Similarly to the case of alkali metals, the critical temperature for the first-order transition gas-liquid obeys the relation

$k T_{c r} \approx 0.1 E_{e x}$.

We demonstrate the table and figures for different quantum-sized structures (of various sizes) and different dimensionality for various semiconductors ( $\mathrm{Si}, \mathrm{GaAs})$ where the energy bonds of excitons $E_{s}$ vs $d$ and $T_{c r}$ vs $d$ show the possibility to realize EHLP even at relatively high temperatures (Fig. 2).

The condensation energy for 2D case looks like [7]:

$E_{\text {cond.s }} \sim\left(\frac{n}{v}\right) \frac{n_{p}}{n_{p}}-a\left(\frac{n}{v}\right)^{1 / 2}-E_{\text {corr.s }}$

( $\alpha$ is a constant). In this case, the greater increase in binding energy is predicted for many-valley $(v>1)$ and strongly anisotropic semiconductors then that for threedimensional one, as shown in Fig. 1 ( $v$ is the number of valleys, index "s" means "surface").

In a general form, in effective mass approximation the problem of QD exciton and electron spectra can be given by the Schrödinger equation (1) with the following potential: $\quad U_{s}(r)+U_{c v}(r)+U_{e h}(r), \quad$ where $U_{s}(r)$ denotes polarization potential, which account the real shape of the interface area; $U_{c v}(r)$ - band barriers; $U_{e h}(r)$ - Coulomb interaction. Effective mass and band coordinate dependence was approximated by the step function (rectangular wall). Eigenvalues were obtained from the following Schrödinger equation:

$\left[-\frac{\hbar^{2}}{2 m^{*}{ }_{e}\left(r_{e}\right)} \Delta_{e}-\frac{\hbar^{2}}{2 m^{*}{ }_{h}\left(r_{h}\right)} \Delta_{h}+U\left(r_{e}, r_{h}\right)\right] \times$

$\times \Psi\left(r_{e}, r_{h}\right)=E \Psi\left(r_{e}, r_{h}\right)$,

where the Coulomb interaction term is:

$U_{e h}(r)=-\frac{e^{2}}{4 \pi \varepsilon(d) \varepsilon_{0}} \int \frac{\left|\Psi\left(r^{\prime}\right)\right|^{2}}{\left|r-r^{\prime}\right|} d^{3} r^{\prime}$.

We use one-dimensional polarization potential term that is suitable for estimation in macroscopic approximation for the dielectric constant:

$U_{s}=\frac{1}{n} \sum_{i=1}^{n} \frac{e^{2}\left(\frac{\varepsilon_{Q D}-\varepsilon\left(x_{i}\right)}{\varepsilon\left(x_{i}\right)}\right)}{4 \pi \varepsilon_{0} \varepsilon_{Q D}\left(\frac{d_{Q D}}{2}+x_{i}\right)}$,

where QD is quantum dot.

To account extension of $\varepsilon\left(x_{i}\right)$, we approximate them by several $(n)$ rectangular steps in the form (8). This equation results from the one-dimensional potential created by the rectangular step. Then, the exciton energy levels can be represented by the following expression $[4,5]$ : 

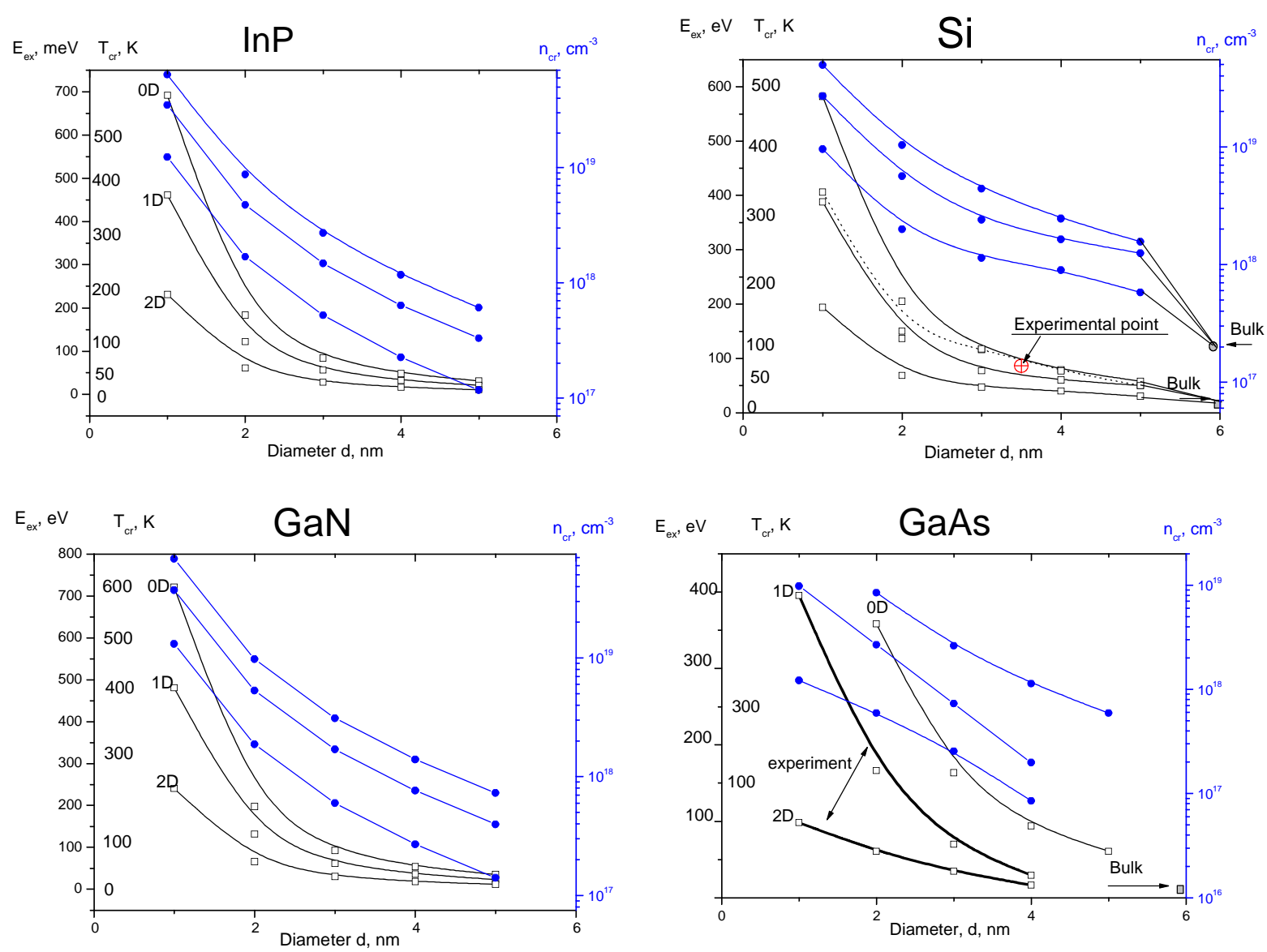

Fig. 2. Calculated curves for the exciton binding energy $E_{e x}$, critical temperature $T_{c r}$ (open symbols), and critical concentration of EHLP $n_{c r}$ (filled symbols) for nanostructures of different dimensionality (0D, 1D, 2D).

$$
\begin{aligned}
& E_{e x}=\frac{-e^{2}}{2 \pi \varepsilon_{e f f}(d) \varepsilon_{0} d} \beta_{n}+\sqrt{\frac{3 \pi \hbar^{2} n^{2} e^{2}}{m_{e x}^{*} d^{3} \varepsilon_{e f f}(d) \varepsilon_{0}}}, \\
& \beta_{n}=2 \int_{0}^{\pi n} \frac{d y \sin ^{2} y}{1-y^{2}} .
\end{aligned}
$$

Instead of the exciton model where the hydrogenlike electron-hole atom with the effective reduced mass $\mu_{e x}^{*}=\frac{m_{e}^{*} m_{h}^{*}}{m_{e}^{*}+m_{h}^{*}}$ is commonly considered, we use another model - hole, oscillating in the field caused by a fastmoving electron (adiabatic oscillator approximation). This approach is preferable for localized states. In the case of exciton localized in QD, this model has minimized mistakes. To describe excitons in quantum dots, one can predict a drastical increase of the binding energy and decrease in the exciton radius. Hence, the Mott approximation converts into Frenkel approximation. In the latter case, one can use the Landau model of "electron atom", for the energy of interaction of a moving electron and hole. In this case, for exciton as oscillator, we use the following relation for the effective excitonic mass: $m_{e x}^{*}=m_{e}^{*}+m_{h}^{*}$. The first term in (4) represents the hydrogen-like Coulomb interaction potential energy; the second term takes into account the relative e-h movement (kinetic) energy. In the effective medium approximation for the dielectric permittivity:

$\varepsilon_{e f f}(d)=\sum_{i} \frac{1}{\varepsilon\left(r_{i}\right)} \cdot \int_{r_{i}}^{r_{i+1}} r^{2}|\Psi(r)|^{2} \cdot d r$.

For estimation, one can use this simple form:

$\frac{1}{\varepsilon_{e f f}(d)}=\frac{1}{\varepsilon_{\mathrm{Si}}} P_{1}(d)+\frac{1}{\varepsilon_{\mathrm{SiO}_{2}}} P_{2}(d)$,

where $P_{1}(d)$ and $P_{2}(d)$ are the weighting factors for QD and surrounding medium,

$$
\int_{0}^{d / 2} r^{2}|\Psi(x)|^{2} d r=P_{1} ; \quad \int_{d / 2}^{\infty} r^{2}|\Psi(x)|^{2} d r=P_{2}
$$

It is necessary to use the normalization requirement:

$$
\int_{0}^{\infty} r^{2}|\Psi(x)|^{2} d r=1
$$

Here, $P_{1}$ and $P_{2}$ are the probabilities to find the particle inside and outside the QD, respectively. The solution of the Schrödinger equation in the case of 
spherical symmetry can be found in the form of spherical Bessel functions:

$\Psi_{1}=B J(k, r)$

(inside the QD),

$\Psi_{2}=A N(\beta, r)$

(outside the QD),

$\beta=\frac{\sqrt{2 m_{2}^{*}(U(r)-E)}}{\hbar}, \quad k=\frac{\sqrt{2 m_{1}^{*} E}}{\hbar}$,

$m_{e 1}^{*}$ and $m_{e 2}^{*}$ are the effective masses of an electron inside and outside of the QD, respectively; $A$ and $B-$ constants that determine the magnitude of the wave function, $E$ - eigenvalue for the ground $s$-orbital state. conditions:

By usual way, using these boundary and initial

$\Psi_{1}(d / 2)=\Psi_{2}(d / 2)$,

$\frac{1}{m_{e 1}^{*}} \frac{d \Psi_{1}(d / 2)}{d r}=\frac{1}{m_{e 2}^{*}} \frac{d \Psi_{2}(d / 2)}{d r}$,

one can obtain the following non-algebraic equation

$\frac{\frac{d \beta}{2}-1}{m_{e 2}^{*}}=\frac{1-\frac{d k}{2} \operatorname{ctg}\left(\frac{k d}{2}\right)}{m_{e 1}^{*}}$,

solution of which with the rule of roots selection $\operatorname{ctg}\left(\frac{d \beta}{2}\right)<0$ gives us the energy levels for electrons.

Similar relation takes place for holes (with the effective masses $\left.m_{e 1}^{*}, m_{e 2}^{*}\right)$. To determine constants $A$ and $B$, it is necessary to use normalization requirement.

For numerous semiconductors, critical concentrations for e-h pairs $\left(n_{c r} \sim r_{B}^{-3}\right)$ and critical temperatures of condensation were predicted [1-5]. We have calculated the values of these characteristics for various quantum-sized structures and semiconductors by using the relation discussed earlier in literature [2-4]:

\begin{tabular}{|c|c|c|c|c|c|c|}
\hline \multicolumn{4}{|l|}{ Bulk } & \multicolumn{3}{|c|}{ Quantum } \\
\hline & $\begin{array}{l}\varepsilon_{e x}, \\
\mathrm{meV}\end{array}$ & $\begin{array}{l}T_{c r}, \\
\mathrm{~K}\end{array}$ & $\begin{array}{l}n_{c r} \\
\mathrm{~cm}^{-3}\end{array}$ & $\begin{array}{l}\varepsilon_{e x}, \\
\mathrm{meV}\end{array}$ & $\begin{array}{l}T_{c r}, \\
\mathrm{~K}\end{array}$ & $\begin{array}{l}n_{c r}, \\
\mathrm{~cm}^{-3}\end{array}$ \\
\hline \multirow[t]{2}{*}{$\mathrm{Si}$} & 15 & 10 & $\begin{array}{l}4 \times 10 \\
18\end{array}$ & $\begin{array}{l}2 \mathrm{D} \\
17\end{array}$ & $\sim 15$ & $\begin{array}{l}\sim 5 \times 1 \\
0^{18}\end{array}$ \\
\hline & & & & $\begin{array}{l}\text { QD } \\
60-140\end{array}$ & $\begin{array}{l}75- \\
150\end{array}$ & $\sim 10^{19}$ \\
\hline GaAs & 3.8 & 4.5 & $\begin{array}{l}2.6 \times \\
10^{16} \\
\end{array}$ & 6.5 & 8.7 & $\begin{array}{l}1.8 \times 1 \\
0^{16}\end{array}$ \\
\hline $\mathrm{GaP}$ & & & & 6.5 & 20 & $\begin{array}{l}\sim 3 \times 1 \\
0^{16} \\
\end{array}$ \\
\hline $\mathrm{CdS}$ & & & $10^{18}$ & & & \\
\hline $\mathrm{ZnO}$ & 60 & 80 & $\begin{array}{l}2.5 \times \\
10^{17}\end{array}$ & $\begin{array}{l}2 \mathrm{D} \\
80\end{array}$ & 110 & $\begin{array}{l}\sim 3 \times 1 \\
0^{15}\end{array}$ \\
\hline InSe & & $\sim 150$ & & & & \\
\hline $\mathrm{Ge}$ & $\begin{array}{l}3.5- \\
4.4 \\
\end{array}$ & 6.6 & $10^{17}$ & & & \\
\hline & & & & & & \\
\hline
\end{tabular}

In Figs 2, we have depicted the resulting data for a set of important semiconductors both with a narrow bandgap, and with moderate and wide ones: InP, Si, $\mathrm{GaAs}$, and GaN. As seen, the calculated characteristics can reach unusualy extreme values - some hundreds of $\mathrm{meV}$ and degrees of Kelvin for the exciton bond energy $E_{e x}$ and critical temperatures $T_{c r}$, respectively, and $10^{19} \mathrm{~cm}^{3}$ - for critical e-h pair concentration already at $1-$ $2 \mathrm{~nm}$ for the QD structures. Below, we will demonstrate the possibility of the new engineering approach to realize a set of unusual applications of the EHLP in nanoelectronics.

\section{Experimental results}

Let us consider first indirect band semiconductors, as an example $\mathrm{Si}$, which represents the case with large values of the lifetime and sufficiently large bond energy of excitons. Recently, obtained results for Si-ultra-thin monocrystalline films up to $50 \mathrm{~nm}$ show that, beside the exciton narrow line of photoluminescence (PL) spectra, at large laser illumination $(2-30 \mathrm{~mW})$ the rather broad PL line close to the energy of the electron band ( $\sim 12 \mathrm{meV})$ have been observed [16,17] (Fig. 3). With increasing the excitation level, the width of this line and energy position occurred constant (Fig. 3), while the intensity increased in accord with the exp power about 2.

For the bulk case, predicted is the power $3[10,17]$. These facts proved appearance of the e-h droplets and influence of the double dimensionality. The exciton energy $17 \mathrm{meV}$ is higher as compared to the bulk one $\sim 15 \mathrm{meV}$ for both films, and the energy of condensation $17.5 \mathrm{meV}$ is also remarkably higher than for the bulk one $\sim 14 \mathrm{meV}$ [17]. Probably, condensation takes place on surfaces of ultra-thin layers, which determines appearance of EHLP at rather low excitation levels $[4,5]$. The influence of surface is also demonstrated by the lower energy of surface LO phonons, which for the bulk $\mathrm{Si}$ $\omega_{L O}^{v} \sim 63 \mathrm{meV}$ is caused by the surface confinement effect. In addition, obtained from the relation (1) and value of $E_{e x}$ the values of the critical temperatures $T_{c r} \sim 15-17 \mathrm{~K}$ are higher than those used in experiment $T \sim 10 \mathrm{~K}$, so appearance of the EHLP is probable.

The following group of experiments is related with luminescent direct-gap semiconductors (GaAs, $\mathrm{ZnO}$, $\mathrm{InSe}$ ) that have rather high exciton binding energy.

As mentioned above, in this case the more favorable experimental conditions to observe EHLP, including surface and nanosize structure, takes place because of intense luminescence. Namely, for such objects with the specially treated surface (cleaning surface, gettering, ion and electron bombardment etc.) to create a space charge attracting the carriers, it was first demonstrated luminescence of the surface bounded excitons that, with increasing of the irradiation power, were transformed into the electron-hole liquid condensate (named as "surface liquid droplets") [15]. 


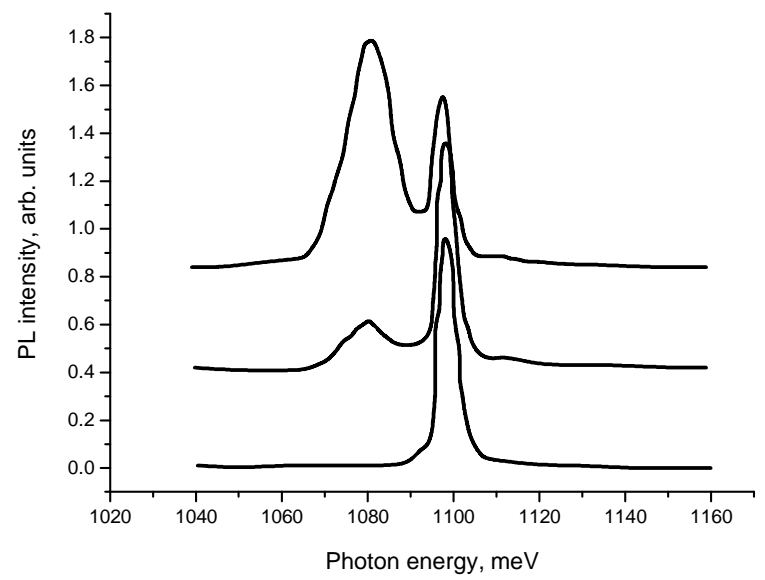

Fig. 3. Photoluminescence spectra with various excitation levels (upper curve corresponds to the highest excitation level).

The very thin $(\sim 0.3 \mu \mathrm{m})$ surface layer of $n$-type $\left(n_{0} \sim\right.$ $\left.10^{17} \mathrm{~cm}^{-3}\right) \mathrm{GaAs}$ with long lifetime $\left(\tau>10^{-6} \mathrm{~s}\right)$ was prepared due to gettering (at $550-600{ }^{\circ} \mathrm{C}$ ) by deposition of an insulator $\mathrm{Si}_{3} \mathrm{~N}_{4}$ film, i.e. by creation of the rather perfect heterostructure $\mathrm{Si}_{3} \mathrm{~N}_{4}$-GaAs [5]. For this thin-layer structure the exciton binding energy was twice increased (from $\sim 3.8$ up to $\sim 6 \mathrm{meV}$ ) due to quasi-dimensionality, and at large excitation in PL spectra the broad liquid plasma luminescence line appears from the red side of the spectrum, being shifted from the exciton line by 3.5 to $5 \mathrm{meV}$. Other signs of EHLP creation are as follows:

1. Constance of the width of PL spectral line $W$, which is in direct proportion to the sum of the Fermi energy for electrons and holes. The latter determines the density of carriers under power excitation (Fig. 4).

2. Narrowing the halfwidth of the line vs temperature $T$, which for Fermi liquid was predicted due to the increasing entropy in the system $S \sim \gamma T$. Here, when $T$ is close to the critical one, the slow decrease of $W$ has been predicted [1-5]:

$$
W \approx W_{0}\left(1-\gamma T^{2}\right) \text {. }
$$

These dependences were observed experimentally (Fig. 5), what allowed to calculate the phase diagram for subband EHLP (gas-liquid) and compare it with the bulk one as demonstrated in Figs. 5 and 6. Also, it is shown a remarkable increase in the stability of Fermi liquid state in GaAs layered structure against temperature (approximately two times increasing from approximately 4 up to $8 \mathrm{~K}$ ) takes place, which is in accord with the relations (4).

For the discussed layered structure $\mathrm{GaAs}-\mathrm{Si}_{3} \mathrm{~N}_{4}$, the predicted $T_{c r}^{v} \sim 4.2 \mathrm{~K}, T_{c r}^{s} \sim 8.5 \mathrm{~K}$, and the energy of condensation to electron-hole liquid, respectively: $n_{v_{T=0}} \approx 2.8 \times 10^{16} \mathrm{~cm}^{-3}, n_{2 D_{T=0}} \approx 1.8 \times 10^{16} \mathrm{~cm}^{-3}$, which is in accord with Mott-transformation criteria: $n_{e h} r_{B}^{-3} \sim 1, \quad n_{e h} r_{B}^{-2} d \sim 1$ for bulk and quasi twodimensional e-h plasma (where $d$ is the thickness of a quantum layer).

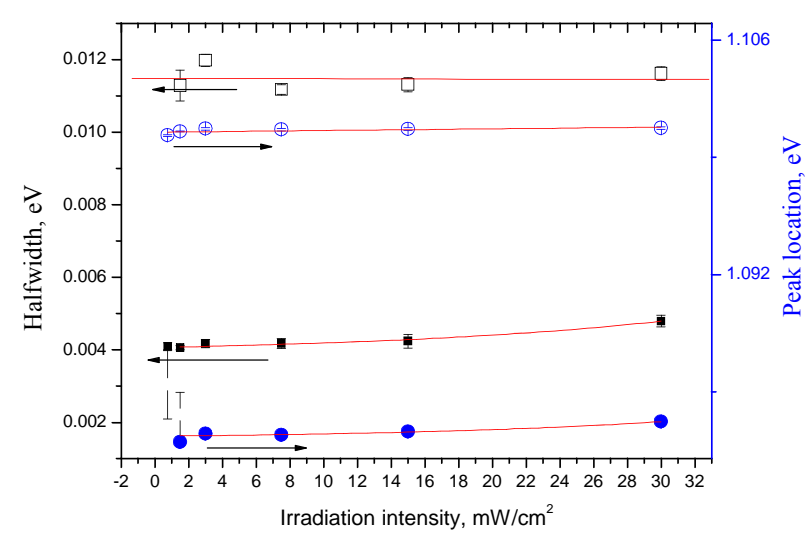

Fig. 4. Basic parameters of PL spectrum of Si thin layer: halfwidth $W$ and energy maximum of e-h liquid luminescence band vs the irradiation intensity. Empty symbols for excitons, filled for e-h liquid. Circles - peak location, squares halfwidth. There two peaks in photoluminescence spectra (Fig. 3) filled symbols correspond to the left peak.

(a)

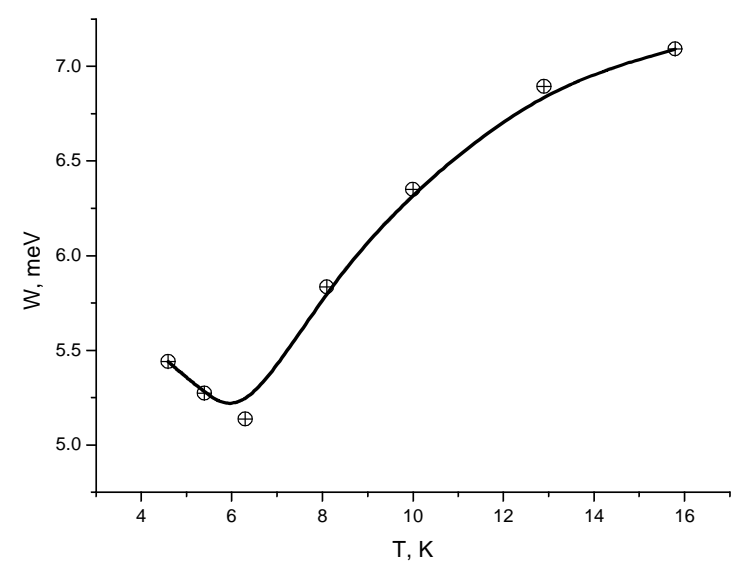

(b)

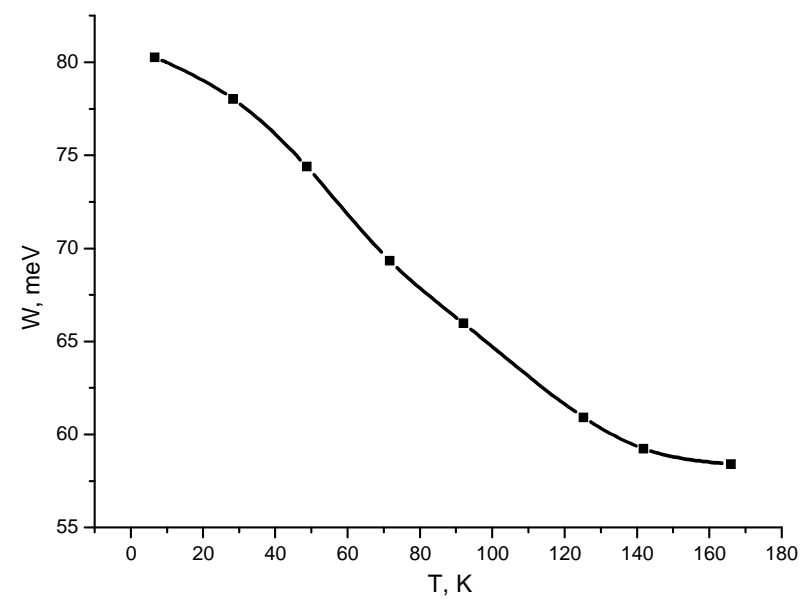

Fig. 5. Non-monotonic temperature dependence of the halfwidth $W$ for the PL peak of EHLP in heterostructures GaAs- $\mathrm{Si}_{3} \mathrm{~N}_{4}$ (a) and layered crystal InSe (b). 


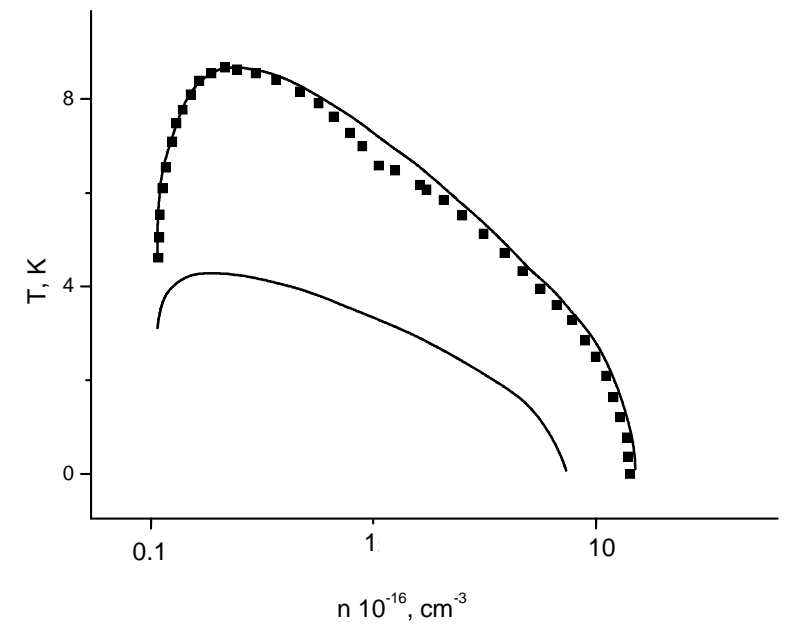

Fig. 6. Phase diagram of two- (upper) and three- (bottom) dimensional e-h liquid for heterostructure GaAs- $\mathrm{Si}_{3} \mathrm{~N}_{4}$. Squares - experimental points.

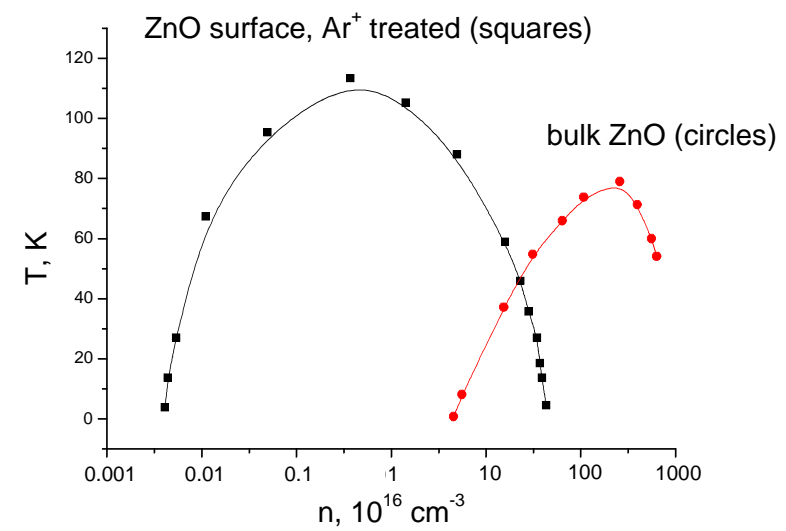

Fig. 7. Phase diagram of two- (left) and three- (right) dimensional e-h liquid.

Values of the obtained density for e-h pairs $n_{c}$ in EHLP $(1 \ldots 2) \times 10^{16} \mathrm{~cm}^{-3}$ for GaAs mean the Fermi degenerated state $\left(N_{e}^{*} \sim 10^{16} \mathrm{~cm}^{-3}\right)$, so, there is a quantum Fermi liquid state of EHLP.

Another luminescent crystal $\mathrm{ZnO}$ is the direct-band semiconductor with a much higher value of the exciton energy $\sim 80 \mathrm{meV}$. To create the liquid e-h plasma on the surface, $\mathrm{Ar}^{+}$bombardment to form the surface exciton trapped centers was performed. Two types of droplets were observed with rather high critical temperature: $T_{c r 1} \sim 100 \mathrm{~K}$ and $T_{c r 2} \sim 80 \mathrm{~K}$ due to different local surface energies. As we assume, these are quasi-2D and quasi$1 \mathrm{D}$ symmetry centers. The critical concentration value is of the order $(1 \ldots 3) \times 10^{17} \mathrm{~cm}^{-3}$ (Fig. 7).

Even higher values of $E_{e x}$ are inherent to the layered semiconductor crystals, for example GaS. It demonstrates large critical temperature higher than $T_{c r}>150 \mathrm{~K}$, e-h concentration $n_{c r} \sim 10^{18} \mathrm{~cm}^{-3}$ (Fig. 5). This semiconductor processes the very large excitation band energy $E_{e x} \sim 100 \mathrm{meV}$, which leads to a high critical temperature of condensation, much higher than the liquid helium temperature, and for quantum-sized structures the critical temperature is predicted to be much higher than the room temperature.

Experimental difficulties appear with increasing the excitation power, which causes overheating and leads to a sharp decrease in the carrier lifetime $\tau$. To avoid this, it is necessary to use short-time pulses $t_{p}$. However, when $t_{p}<<\tau$ the criteria of condensation cannot be realized. This situation is typical for direct-gap semiconductors and superlattices (like GaAs, $\mathrm{GaP}$, and $\mathrm{GaN}$ ) [19, 22].

\section{Conclusion}

In the quantum-sized low-dimensional (nanosize) semiconductor structures, the properties of EHLP have essential features, which make EHLP more stable, critical concentration lower and $T_{c r}$ higher than in the bulk. These mechanisms can be realized in nanostructures prepared by recently developed high technologies (like to molecular beam epytaxy and synergetic).

For ultrasmall QDs $\left(d<<r_{B}^{*}\right)$, when the number of free carriers and excitons are too low for collective interaction and EHLP creation, instead of it, probable formation of quasi-particles - trions, poliexcitons under a high excitation level of carriers (as opposite to bulk) takes place. And only in the case of large concentrations of quantum dots, creation of plasma is possible due to tunnel interaction between neighbour clusters. This case will be analyzed separately.

\section{Acknowledgements}

This work was partially done in the frame of Project NanoPhysics, NAS of Ukraine and Project M/175-2007. We are thankful to Prof. D.V. Korbutyak, Prof. S.V. Krylyuk and Prof. V.I. Sugakov for discussions.

\section{References}

1. C. Jeffries and L. Keldysh, Electron-Hole Droplets in Semiconductors. North-Holland, Amsterdam, 1983.

2. T. Rice, J. Hensel, T. Phillips, G. Thomas, The Electron-Hole Liquid in Semiconductors. Academic Press, inc., 1977.

3. E.A. Andryushin, Energy of ground state of layered ED-liquid // Fizika tverdogo tela 18(9), p. 24932498 (1976), in Russian.

4. V.G. Litovchenko, Fundamentals of Physics of Semiconductor Layered Systems. Kiev, Naukova dumka, 1980 (in Russian).

5. V.G. Litovchenko, D.V. Korbutyak, S.V. Krylyuk, Yu.V. Kryuchenko, Non-equilibrium quantum dimensional effects at the surface of 
semiconductors and in thin-film layers // Ukrainsky fizychny zhurnal 43(11), p. (1998), in Ukrainian.

6. A.V. Larionov, V.B. Timofeev, Collective behaviour of interlayer excitons in GaAs/AlGaAs double quantum layered structures // Pis'ma v Zhurnal Eksperim. Teor. Fiziki 71, p. 174-181 (2000), in Russian.

7. V.N. Dobrovolsky, V.G. Litovchenko, Surface Electronic Transport in Semiconductors. Oxford University Press, 1991.

8. O.V. Nazarenko, V.I. Sugakov, Study of the exciton condensed phase in 2D systems // Ukrainsky fizychny zhurnal 49(10), p. 1029-1033 (2004), in Ukrainian.

9. L.V. Keldysh, A.P. Silin, Condensation of excitons in direct semiconductors // Zhurnal Eksperim. Teor. Fiziki 69(3), p. 1053-1065 (1975), in Russian.

10. W.F. Brinkman and T.N. Rice, Liquid in semiconductors // Phys. Rev. 7, p. 1508 (1973).

11. E.A. Andryushin, A.P. Silin, Two-dimensional EDliqiud // Fizika tverdogo tela 18(8), p. 2132-2138 (1976), in Russian.

12. L.V. Keldysh, Collective properties of excitons in semiconductors // Excitons in semiconductors №1, p. 5-22 (1971).

13. Y. Kuzamoto, H. Kamimura, Theory of 2dimensional ED-liquids, application to layer type semiconductors // J. Phys. Soc. Jpn. 37(3), p. 716721 (1974).

14. D.V. Korbutyak and V.G. Litovchenko, Expansion of the electron-hole liquid plasma at GaAs surface // Phys. status solidi (b) 120(87), p. 87-97 (1983).

15. D.V. Korbutyak, Yu.V. Kryuchenko, V.G. Litovchenko, R. Baltrameyunas, E. Gerazimas, and
E. Kuokshtis, Investigation of the optical gain spectra in two-dimensional quantum well heterostructures // Zhurnal Eksperim. Teor. Fiziki 96, p. 1332-1339 (1989).

16. V.G. Litovchenko, D.V. Korbutyak, and Yu.V. Kryuchenko, Investigation of the collective properties of excitons in polar semiconductors (ZnO) // Zhurnal Eksperim. Teor. Fiziki 54(6), p. 1093-1099 (1981), in Russian.

17. S. Nihonyanagi, Y. Kanemitsu, Enhanced luminescence from electron-hole droplets in silicon nanolayers // Appl. Phys. Lett. 85(23), p. 5721 (2004).

18. V.G. Litovchenko, D.V. Korbutyak, and Yu.V. Kryuchenko, Radiative properties of nonequilibrium long-wavelength plasmons in an electron-hole plasma // Sov. Phys. Solid State 29(3), p. 454-456 (1987).

19. W. Langbein, S. Hallstein, H. Kalt, R. Notzel, and K. Ploog, Negative-differential band-gap renormalization in type-II GaAs/AlGaAs superlattices // Phys. Rev. B 51(3), p. 1946-1949 (1995).

20. D.W. Wang and S. Das Sarma, Many-body effects on excitonic optical properties of photoexcited semiconductor quantum wire structures // Phys. Rev. B 64, 195313 (2001).

21. P. Denk, J.L. Pelouard, Temperature-dependent resistivity anomaly in doped AlAs/GaAs lateral superlattices: Evidence for Fermi-liquid behavior // Phys. Rev. B 63, 041304(4) (2001).

22. Yu.E. Lozovik, O.L. Berman and M. Willander, Superfluidity of indirect excitons and biexcitons in coupled quantum wells and superlattices // J. Phys.: Condens. Matter. 14, 12457-12475 (2002). 